\title{
Polypeptide Profiles of Diminazene Aceturate Resistant Trypanosoma evansi Organisms Isolated from a Buffalo
}

\begin{abstract}
Keywords: Buffaloes; Diminazene aceturate; India; SDS Page; Trypanosomosis

Abstract

Trypanosomosis was recorded in a buffalo during the month of September 2014 in Proddatur of Andhra Pradesh, India. Disease was confirmed by the presence of Trypanosoma evansi organisms in peripheral blood smears. Buffalo was treated with intra muscular administration of diminazene aceturate at the rate of $3.5 \mathrm{mg}$ per $\mathrm{kg}$ body weight along with supportive therapy. After two days of therapy, re-examination of blood smear revealed the presence of live Trypanosoma evansi organisms. To determine the profiles of diminazene resistant Trypanosoma evansi organism's whole blood was collected and processed. Whole cell lysate antigen was prepared from the host cell free Trypanosoma evansi parasites. A total of 8 polypeptide bands with relative molecular weight of $34,48,53,55,64$, 74,80 and $98 \mathrm{kDa}$ were identified. Buffalo was successfully treated with sub cutaneous administration of antrycide prosalt, at the dose rate of $7.5 \mathrm{mg}$ perkg body weight along with supportive therapy.
\end{abstract}

\section{Introduction}

Trypanosomosis is an important haemoprotozoan disease of domesticated animals. Among the several species of trypanosomes, Trypanosoma evansi is the most common species in India. It causes anaemia, lowering milk yield which leads to economic losses to the small farmers [1]. Cattle and buffaloes are considered as reservoir of parasite for other domestic as well as pet animals. Due to stress reservoir hosts may also suffer with clinical trypanosomosis [2]. In India, diminazene aceturate, isometamidium chloride and quinapyramine sulphate and chloride are currently available drugs for the treatment of trypanosomosis [3]. Diminazene aceturate is one of the most common drugs used in the treatment of trypanosomosis and babesiosis in animals. Previous studies shown that, diminazene and isometamidium are therapeutically effective against clinical T. evansi infection [4]. Consistent use of low doses of diminazene aceturate leads to the development of resistant strains of trypanosome species and relapses of infection was observed few days of post treatment [5]. Hence present study was conducted to record the protein profiles of diminazene aceturate resistant Trypanosoma evansi organisms.

\section{Materials and Methods}

A 9 nine years old milch buffalo with history of anorexia, chronic emaciation, reduction in milk yield and occasional excitement (Figure 1) was referred to the Teaching Veterinary Clinical Complex, College of Veterinary Science, Proddatur during the month of September, 2014. Up on clinical examination, buffalo showed pale mucus membranes, corneal opacity (Figure 2), pyrexia $\left(103.2^{\circ} \mathrm{F}\right)$, heart rate of $82 \mathrm{bpm}$, respiratory rate of $26 / \mathrm{min}$, enlarged sub mandible lymph nodes and oedema of the fore limbs. Electrocardiography and examination with metal detector was carried out to detect other abnormalities
Journal of

Veterinary Science \& Medicine

\section{S. Sivajothi ${ }^{1 *}$ and B. Sudhakara Reddy ${ }^{2}$}

${ }^{1}$ Department of Veterinary Parasitology, College of Veterinary Science, Proddatur 516360, Sri Venkateswara Veterinary University, Andhra Pradesh, India.

${ }^{2}$ Teaching Veterinary Clinical Complex (Veterinary Medicine), College of Veterinary Science, Proddatur 516360, Sri Venkateswara Veterinary University, Andhra Pradesh, India

*Address for Correspondence

S. Sivajothi, College of Veterinary Science, Proddatur, Sri Venkateswara Veterinary University, Andhra Pradesh, India, E-mail: sivajothi579@gmail.com

Submission: 25 April, 2016

Accepted: 20 June, 2016

Published: 25 June, 2016

Copyright: (๑) 2016 Sivajothi S and Reddy BS. This is an open access article distributed under the Creative Commons Attribution License, which permits unrestricted use, distribution, and reproduction in any medium, provided the original work is properly cited.

Reviewed \& Approved by: Dr. Margarita G. Papazahariadou, School of Veterinary Medicine, Aristotle University of Thessaloniki, Greece

[6-8]. Whole blood, peripheral blood smears, serum, urine and dung samples were collected for laboratory analysis according to the previous studies [9]. Peripheral blood smear revealed the presence of live Trypanosoma spp. organisms. Giemsa stained blood smears confirmed the morphology of Trypanosoma evansi organisms (Figure 3). Initially buffalo was treated with intra muscular administration of diminazene aceturate at the dose rate of $3.5 \mathrm{mg} / \mathrm{kg}$ body weight along with fluid and supportive therapy [10,11]. Diagnosis of Trypanosoma evansi organisms was done by the wet blood film examination and it was confirmed by the examination of stained blood smear [12].

After two days of therapy partial improvement in the condition was noticed. Again blood samples were collected for laboratory analysis and which was positive for live Trypanosoma evansi organisms. Whole blood with addition of EDTA was collected for further studies. Multiplication of Trypanosoma evansi was done in a rat and host cell free Trypanosoma evansi parasites was separated by anion-exchange chromatography using a Diethylaminoethyl cellulose column with phosphate buffer saline glucose solution. The whole

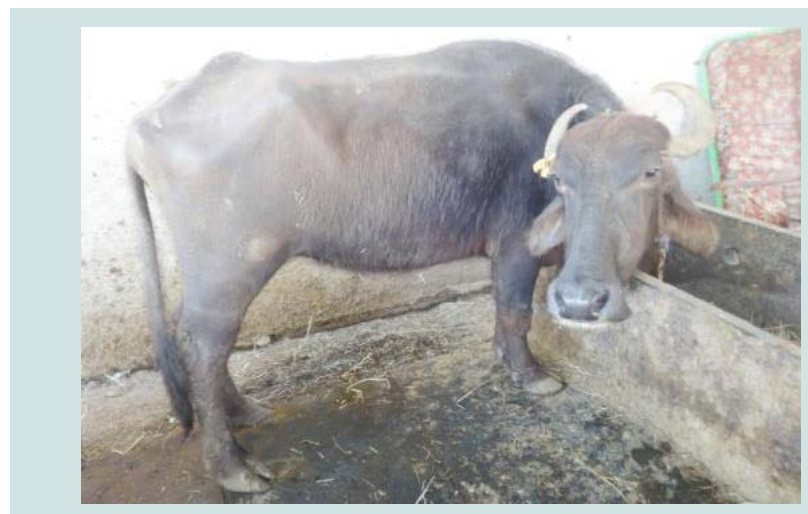

Figure 1: Buffalo suffering with clinical Trypanosomosis. 


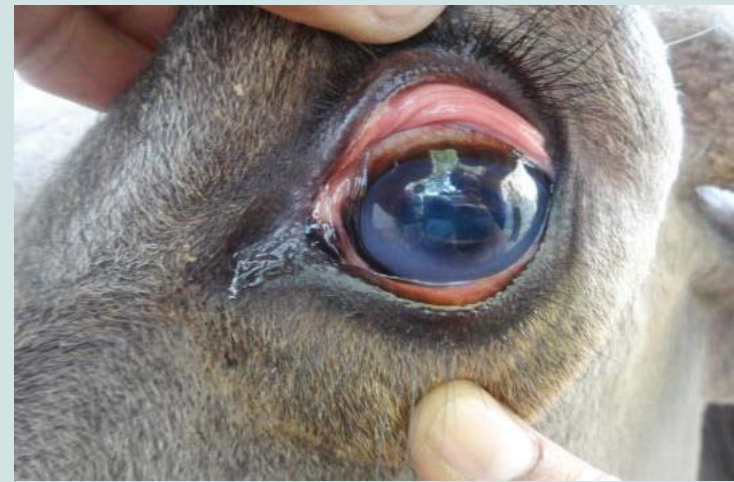

Figure 2: Presence of corneal opacity.

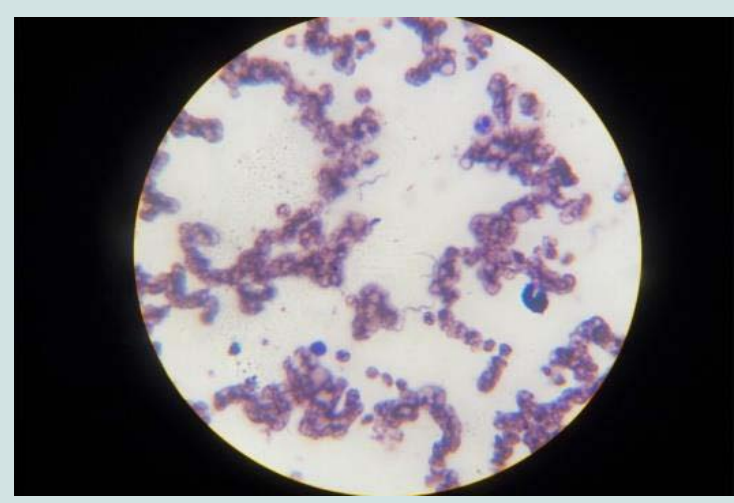

Figure 3: Presence of Trypanosoma evansi organism in stained blood smears

cell lysate antigen was prepared from the parasites and its protein concentration was adjusted to $1.0 \mathrm{mg} / \mathrm{mL}$ in PBS. Polypeptide profile of WCL of T. evansi was determined by SDS-PAGE according to the previous studies $[13,14]$. Buffalo was again treated with sub cutaneous administration of antrycide prosalt at the dose rate of $7.5 \mathrm{mg} / \mathrm{kg}$ body weight along with supportive therapy [15].

\section{Results and Discussions}

After two days of therapy with antrycide prosalt buffalo was free from live Trypanosoma evansi organisms and improvement in the condition was noticed which indicates the presence of diminazene aceturate resistant Trypanosoma evansi organisms in the present case.

Polypeptide profiles of whole cell lysate antigens from the diminazene aceturate resistant Trypanosoma evansi was shown in Figure 4 . In the present study a total of 8 polypeptides were observed when resolved in 10\% SDS-PAGE and stained with Commassie brilliant blue. The approximate molecular weights of these polypeptides were ranged from 34 to $98 \mathrm{kDa}$ with relative molecular weight of $34,48,53,55,64,74,80$ and $98 \mathrm{kDa}$. Whole cell lysate antigen of T. evansi isolate was prepared according to the previous studies [2]. Recorded polypeptide profiles of diminazene aceturate resistant isolates of T. evansi revealed the presence of different bands from the previous studies which indicates the presence of variations in the isolates. Previously 8 polypeptide bands size of $25,34,37,42$, $53,60,74$ and $85 \mathrm{kDa}$ recorded from isolate of cattle. Similarities between the cattle isolate and present isolate was noticed at the band size of 34, 53 and $64 \mathrm{kDa}$ molecular sizes [2].

Previously Laha et al. recorded the 11 dominant polypeptide bands with relative molecular weight ranging from 95 to $13 \mathrm{kDa}$. Among the 11, seven were major polypeptides with relative molecular weight ranges between 86-87, 74-75, 61-62, 51-53, 39, 34-35, $13 \mathrm{kDa}$ and four minor polypeptides ranges 93-95, 46-47, 28-29, 25-26 kDa appeared as common to all T. evansi of different hosts origin [16].

Loss of sensitivity of a trypanosome to a drug which it had previously been susceptible is called as trypanocidal drug resistance. There is increase in the resistance of trypanosomes to the regularly used trypanocidal drugs in animals. Lack of appropriate usage and under dosing is the main predisposing factors for the development of drug resistance [17]. Diminazene aceturate was used as both trypanocidal and babesiacidal drug for domestic livestock. Because of rapidly excreted activity of Diminazene aceturate it is recommended only for therapeutic use. Diminazene binds to trypanosomal kinetoplast DNA and inhibits the synthesis of RNA primers, resulting in accumulation of replicating intermediates, there by inhibiting kDNA replication [18]. According to the previous studies, reduction in the number of trypanosomes immediately after treatment and complete clearance of trypanosomes can be noticed within eight hours of treatment with diminazene aceturate [19].

According to the previous studies, trypanosomes have developed significant resistance to the regularly used diminazene aceturate and isometamidium chloride [3]. In the present study, buffalo had the history of multiple injection of diminazene aceturate whenever suffering with non specific fever. Therapeutic dose rate of diminazene aceturate was given at the dose rate of 3.5 to $7 \mathrm{mg} / \mathrm{kg}$ by intramuscular route. But, often use dose of $3.5 \mathrm{mg} / \mathrm{kg}$ to get rid of the clinical signs, but is most likely unable to cure the infection [11]. In Japan, acquired resistance to diminazene aceturate in a cloned isolate of Trypanosoma evansi [15].

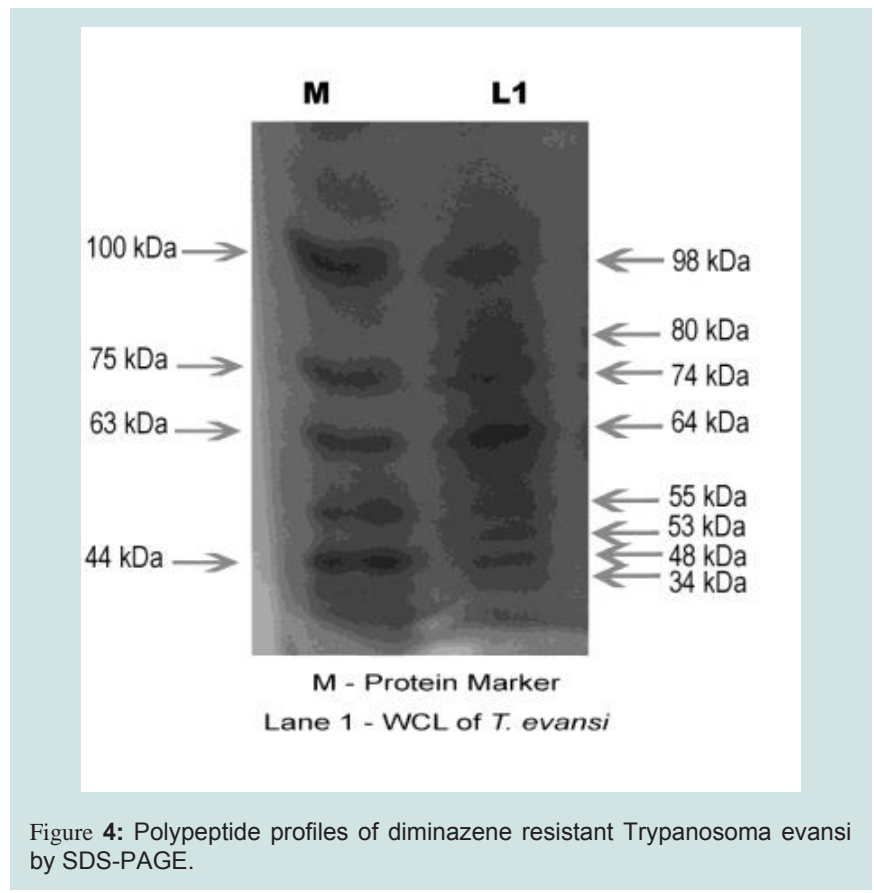


Citation: Sivajothi S, Reddy BS. Polypeptide Profiles of Diminazene Aceturate Resistant Trypanosoma evansi Organisms Isolated from a Buffalo. J Veter Sci Med. 2016;4(1): 1

ISSN: $2325-4645$

Contrast to the present study, in Nigeria self limiting phenomena was recorded in West African dwarf goats infected with Sokoto (Northern Nigeria) isolate of Trypanosoma evansi [20]. But in African countries, animal trypanocidal drug resistance has been reported and they were developed some new molecular detection tools enabling faster diagnosis of drug resistance [21]. Extensive use of the same trypanocidal drug results in appearance of trypanosome strains resistant to the drugs. Previously, in the Mindanao area of Philippines resistance to isometamidium was noticed and its therapeutic dose was increased to $20 \mathrm{mg} / \mathrm{kg}$ from its normal curative dosage $(10 \mathrm{mg} / \mathrm{kg})$. Dose rate of diminazene was also increased in the present country to $10 \mathrm{mg} / \mathrm{kg}$ [22].

In the present study, recorded diminazene aceturate resistant T. evansi might be due to chronic misuse of diminazene aceturate. Diminazene aceturate resistant T. evansi organisms were collected to record the antigenic variation by the parasites from other T. evansi collected in this geographical region. Significant difference was noticed at the $98 \mathrm{kDa}$ molecular weight size. Present study was useful for the characterization of antigenic profile of diminazene aceturate resistant T. evansi and to record the antigenic variations in India. In India recently reported the diminazene aceturate resistant $T$. evansi in a farm. But, no other study was conducted on the diminazene aceturate resistant T. evansi organisms [23].

Previously some of the authors isolated the Trypanosoma evansi organisms from cattle, camel and identified the polypeptide profiles of isolates with molecular weight ranging from 195 to $26 \mathrm{kDa}$ from cattle isolates and molecular weight ranging from 180 to $24 \mathrm{kDa}$ from camel isolates [24]. By conducting the Western blotting, different polypeptides of $T$. evansi with molecular weight ranging from 74 to $38 \mathrm{kDa}$ was identified in experimental animals include bovines, donkeys, dogs and coatis. Among the all 48-46 and $38 \mathrm{kDa}$ bands were mainly recognized in chronic phase of infection. The antigen with apparent molecular weight of $66 \mathrm{kDa}$, was revealed by antibodies from all experimental animals. According to their study, $48-46 \mathrm{kDa}$ polypeptide was identified by antibodies from all naturally infected animals [25]. In the present study, identified the $48 \mathrm{kDa}$ molecular weight polypeptide indicative of chronic type of infection and molecular weight $64 \mathrm{kDa}$ was noticed which was common in all the T. evansi infected animals.

Diamidines molecules bind to the minor groove of DNA at AT-rich sites. They exert their biological activity by primarily binding to DNA and then inhibiting one or more of the DNA dependent enzymes or by directly impeding the transcription process. The selectivity of diamidines is primarily due to the selective accumulation by the pathogen rather than by host cells. Diminazene aceturate do not cross the blood-brain barrier [26]. Diamidines are actively taken up by transporters; alterations of the transporters can cause development of drug resistance. There are numerous reports of resistance to diminazene aceturate in different countries and in several Trypanosoma species. In any case, resistance seems to be limited to highly endemic areas where the use of this drug is very common. Cross resistance to diminazene aceturate with isometamidium and pentamidine have been reported [27]. Drug resistance to diminazene aceturate is less widespread than isometamidium, but increasingly there are reports of multiple drug resistance. Importance of the P2- type purine transporter in the uptake of arsenical diamidines by $T$. evansi and the consequences of inhibition was described and a novel gene, TeDR40, might be a factor contributing to high diminazene aceturate-resistance in T. evansi.

\section{Conclusion}

In conclusion, the Trypanosoma evansi isolate was resistant to diminazene aceturate and showed the different protein profile when compare with the previous studies.

\section{References}

1. Reid SA (2002) Trypanosoma evansi control and containment in Australasia Trends Parasitol 18: 219-224.

2. Sivajothi S, Rayulu VC, Reddy BV, Malakondaiah P, Sreenivasulu D, et al. (2014) Polypeptide profiles of South Indian isolate of Trypanosoma evansi. J Parasit Dis: 1-4.

3. Ukwueze SC, Anne BM, Jibike GI (2012) Trypanocidal efficacy of diminazene in diabetic rats. Iraqi J Vet Sci 26: 33-38.

4. Joshi SS, Singh B (2000) Evaluation of therapeutic and chemo prophylactic efficacy of certain drugs against clinical surra in buffaloes. Indian Vet $\mathrm{J} 77$ : 895-897.

5. Tuntasuvan D, Jarabrum W, Viseshakul N, Mohkaew K, Borisutsuwan S, et al. (2003) Chemotherapy of surra in horses and mules with diminazene aceturate. Vet Parasitol 110: 227-233.

6. Reddy BS, Venkatasivakumar R, Reddy LS, Vani S, Sivajothi S (2015) Analysis of base apex lead electrocardiograms of adult buffaloes. J Dairy Vet Anim Res 2: 00058

7. Reddy LS, Reddy BS, Naik BR, Prasad CS (2014) Haematological and clinical alterations with traumatic reticuloperitonitis in cattle. Int $\mathrm{J}$ Vet Sci 3: 203-205.

8. Sivajothi S, Reddy BS (2014) Brisket oedema due to microfilariosis in a buffalo. Res Rev: J Vet Sci Techno 3.

9. Sivajothi S, Rayulu VC, Reddy BS (2015) Rapid serodiagnosis of Trypanosoma evansi in dogs by colloidal dye immunobinding assay. Comp Clin Pathol 24: 1497-1500.

10. Da Silva AS, Zanette RA, Wolkmer P, Costa MM, Garcia HA, et al. (2009) Diminazene aceturate in the control of Trypanosoma evansi infection in cats. Vet Parasitol 165: 47-50.

11. Desquesnes M, Gutierrez C (2011) Animal trypanosomosis: an important constraint for livestock in tropical and subtropical regions. In: Javed MT (Ed). Livestock: rearing, farming practices and diseases, Nova Science Publishers, New York, pp. 127- 144.

12. Reid SA, Husein A, Copeman DB (2001) Evaluation and improvement of parasitological tests for Trypanosoma evansi infection. Vet Parasitol 102: 291-297.

13. Laemmli UK (1970) Cleavage of structural proteins during the assembly of the head of bacteriophage T4. Nature 227: 680-685.

14. Sivajothi S, Rayulu VC, Reddy BS, Reddy BV (2015) Polypeptide profiles of whole cell lysate antigens of Trypanosoma evansi isolated from the dogs by SDS-PAGE. J Dairy Vet Anim Res 2: 00047

15. Witola WH, Tsuda A, Inoue N, Ohashi K, Onuma M (2005) Acquired resistance to berenil in a cloned isolate of Trypanosoma evansi is associated with upregulation of a novel gene, TeDR40. Parasitology 131(Pt 5): 635-646.

16. Laha R, Sasmal NK, Bandyopadhyay S, National Research Center for Protozoan Diseases (2008) Comparative polypeptide profiles of whole cell lysate antigens of Trypanosoma evansi isolated from three different hosts of eastern India. J Protozool Res 18: 11-16.

17. Chitanga S, Marcotty T, Namangala B, Van den Bossche $P$, Van Den Abbeele $J$, et al. (2011) High prevalence of drug resistance in animal trypanosomes without a history of drug exposure. PLoS Negl Trop Dis 5: e1454. 
Citation: Sivajothi S, Reddy BS. Polypeptide Profiles of Diminazene Aceturate Resistant Trypanosoma evansi Organisms Isolated from a Buffalo. J Veter Sci Med. 2016;4(1): 1

ISSN: $2325-4645$

18. Brack C, Delain E (1975) Electron-microscopic mapping of AT-rich regions and of E. coli RNA polymerase-binding sites on the circular kinetoplast DNA of Trypanosoma cruzi. J Cell Sci 17: 287-306.

19. Mitchell JB (1990) Protein conjugates as trypanocide delivery systems. In: Peregrine AS (Ed). Chemotherapy for trypanosomiasis: proceedings of a workshop held at ILRAD, Nairobi, Kenya, 21-24 August, 1989. The International Laboratory for Research on Animal Diseases, Nairobi.

20. Ogbaje CI, Lawal IA, Ajanusi OJ (2011) Infectivity and pathogenicity of Sokoto (Northern Nigeria) isolate of Trypanosoma evansi in West African Dwarf Goats, Int J Anim Vet Adv 3: 117-124.

21. Delespaux V, Chitanga S, Geysen D, Goethals A, van den Bossche $P$ et al. (2006) SSCP analysis of the P2 purine transporter TCOAT1 gene of Trypanosoma congolense leads to a simple PCR-RFLP test allowing the rapid identification of diminazene resistant stocks. Acta Trop 100: 96102.

22. Macaraeg BB, Lazao JV, Abes NS, Mingala CN (2013) In-vivo assessment of the effects of trypanocidal drugs against Trypanosoma evansi isolates from Philippine water buffaloes (Bubalus bubalis). Veterinarski Arhiv 83: 381-392.

23. Ponnudurai G, Sivaraman S, Rani N, Veerapandian C (2015) An outbreak of trypanosomosis in buffaloes caused by diminazene resistant Trypanosoma evansi. Buffalo Bulletin 34: 1-4.

24. Pareek RK, Pathak KM, Kumar S, Garg R, Kapoor M (1999) Polypeptide profile of Trypanosoma evansi isolates of camel and cattle origin. J Vet Parasitol 13: 53-54

25. Aquino LP, Machado RZ, Lemos KR, Marques LC, Garcia MV, et al (2010) Antigenic characterization of Trypanosoma evansi using sera from experimentally and naturally infected bovines, equines, dogs, and coatis. Rev Bras Parasitol Vet 19.

26. Soeiro MN, De Souza EM, Stephens CE, Boykin DW (2005) Aromatic diamidines as antiparasitic agents. Expert Opin Investig Drugs 14: 957-972.

27. Witola WH, Inoue N, Ohashi K, Onuma M (2004) RNA-interference silencing of the adenosine transporter-1 gene in Trypanosoma evansi confers resistance to diminazene aceturate. Exp Parasitol 107: 47-57.

\section{Acknowledgements}

Authors are thanking full to the authorities of Sri Venkateswara Veterinary University for providing the facilities. Corresponding author expressed her special thanks to the Mr. S. Jagadeeswar Reddy who helped me a lot during the study period. 